\section{HISTORICAL}

NEUROSCIENCE

\title{
Charcot et Babinski: au-delà de la simple relation professeur-élève
}

\author{
Rami Massie
}

\begin{abstract}
RÉSUMÉ: Jean-Martin Charcot (1825-1893) est reconnu aujourd'hui comme le père de la neurologie clinique en France. Il y forma toute une génération de neurologues proéminents parmi lesquels Joseph Babinski avec qui il eut une relation particulière. Babinski fut sans aucun doute l'élève préféré de Charcot et leur collaboration à la Salpêtrière était excellente. Les deux hommes se vouaient énormement de respect mais il est malheureux que cette relation ait pu, en une occasion particulière, nuire à Babinski. L'agrégation lui fut en effet refusée par Bouchard probablement à cause de celle-ci. Ce refus eut des conséquences futures sur les deux hommes. Malgré cela, le neurologue d'origine polonaise garda une admiration sans borne pour son maître même lorsqu'il continua les recherches de Charcot sur l'hystérie après sa mort. Il en vint à contredire ce dernier sur plusieurs points fondamentaux sans que cela ne diminue en rien son dévouement envers lui. Le lien qui unit les deux hommes peut ainsi être vu bien audelà de la simple relation professeur-élève et pourrait être plutôt comparé à un lien père-fils ce qui n'est pas sans rappeler le modèle original d'enseignement hippocratique.
\end{abstract}

\begin{abstract}
Charcot and Babinski: beyond a simple teacher-student relationship. Jean-Martin Charcot (18251893) is now considered to be the father of clinical neurology in France. He trained a generation of eminent neurologists, among them Joseph Babinski, with whom he had a special relationship. Babinski was undoubtedly Charcot's favorite pupil and they enjoyed an excellent collaboration at la Salpétrière. Even though both men felt tremendous respect for each other, it is sad that this relationship may, in one instance, have been detrimental to Babinski. This is probably the reason why Bouchard denied him full professorship, a decision with eventual consequences for both men. In spite of this, the neurologist of Polish origin held his master in tremendous admiration, even as he pursued Charcot's research on hysteria after his death. Even though Babinski eventually contradicted his master on many fundamental issues, it did not affect his devotion to him. The relationship between the two men can be considered as more than a simple relationship between a teacher and his pupil and may be compared to a father-son relationship, which is a reminder of the original model of Hippocratic teaching.
\end{abstract}

Can. J. Neurol. Sci. 2004; 31: 422-426

Le dix-neuvième siècle en Europe fut une période extrêmement riche dans le domaine du développement intellectuel et scientifique et la jeune discipline de la neurologie n'échappa pas à cette effervescence de la recherche. En France, en particulier, une figure domine toutes les autres et s'impose comme la référence en matière de sciences neurologiques, tant pour ce qui est de l'enseignement que de sa contribution scientifique. Jean-Martin Charcot (1825-1893) (Figure 1) était en effet reconnu internationalement, et on le considère encore aujourd'hui comme le père de la neurologie clinique en France. ${ }^{1}$ Il attirait dans son hôpital de la Salpêtrière des savants réputés venus du monde entier pour écouter ses célèbres Leçons $d u$ mardi et y formait parmi les meilleurs internes de France. À examiner la liste de ses étudiants, c'est à se demander quel grand neurologue français ne fut pas de ses élèves. Parmi tous ces médecins qui allaient plus tard obtenir une réputation internationale, il en est un qui eut avec Charcot une relation privilégiée. Joseph Babinski (1857-1932) (Figure 2) est probablement l'une des personnalités dont le nom restera marqué dans la mémoire de tous les étudiants en médecine, du fait du signe célèbre qu'il a décrit. ${ }^{2}$ Chef de clinique à la Salpêtrière sous Charcot, il est parfois considéré comme l'un des élèves préférés de ce dernier, et eut à en payer le prix lorsqu'il se présenta quelques années plus tard au concours de l'agrégation. Cependant, Babinski fut aussi celui qui eut une des plus grandes influences sur son maître et modifia substantiellement ses vues sur l'hystérie.

\section{Charcot et Babinski à la Salpêtrière}

Jean-Martin Charcot fut un écrivain prolifique. Non seulement ses Euvres Complètes regroupent-elles une grande

From the Université McGill, Montréal, Québec, Canada.

ReCeIVEd November 21, 2003. ACCEPTEDIN FinAlForm MarCh 11, 2004. Reprint requests to: Rami Massie, 6301 Northcrest Place \#11P, Montréal, Québec H3S 2W4 Canada. 


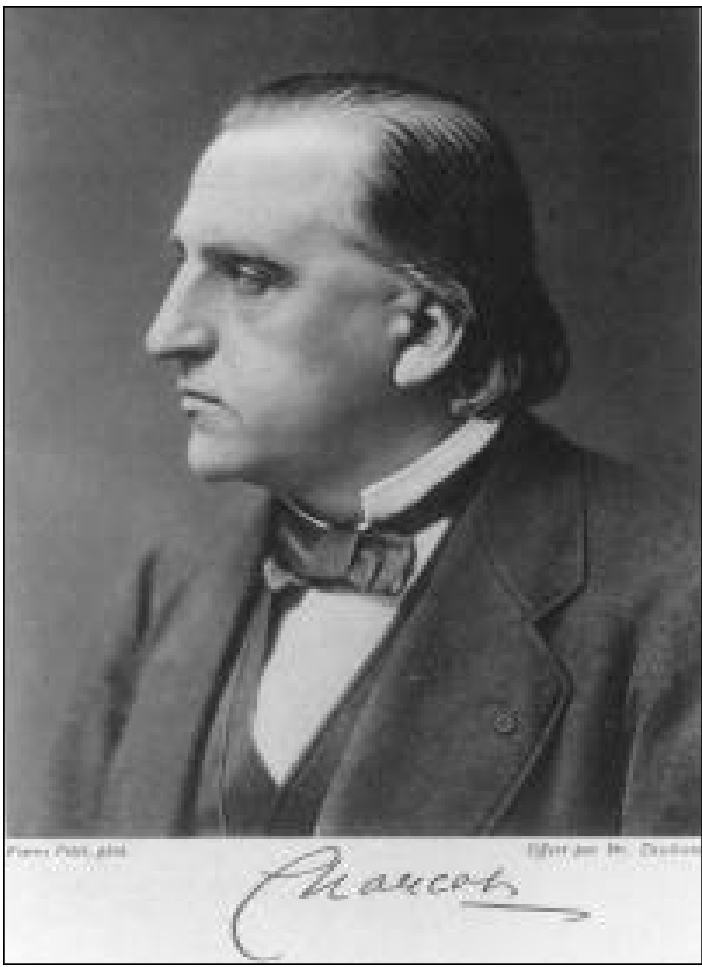

Figure 1: Jean-Martin Charcot (1825-1893).

From the Osler Library of the History of Medicine, McGill University, Montreal

partie de ses observations cliniques et de son enseignement, mais s'y ajoute aussi une volumineuse correspondance entretenue avec de nombreux scientifiques de son époque. Babinski, quant à lui, ne publia pas autant que son maître. Il «goûtait peu les gens qui publient à tort et à travers» et pesait chacun de ses mots avec parcimonie, du fait de «son désir artistique de perfection.» ${ }^{3}$ Malheureusement, aucun des deux neurologues ne coucha par écrit le récit de leur première rencontre, contrairement à ce que firent plusieurs de leurs étudiants dans leurs œuvres commémoratrices. Ainsi, l'on ne peut qu'essayer d'imaginer la scène de cette première rencontre entre les deux hommes en se basant sur leur statut à l'époque.

Joseph Babinski devint chef de clinique à la Salpêtrière à l'automne 1885 alors qu'il n'avait pas encore vingt-huit ans. Il occupa ce poste pendant deux ans, puis resta aux côtés de Charcot jusqu'en 1895, date à laquelle il alla travailler à la Pitié où il fonda son propre service de neurologie. ${ }^{4}$ Contrairement à la plupart des chefs de clinique avant lui, Babinski n'avait pas été interne sous la responsabilité de Charcot à la Salpêtrière. Il avait été reçu interne provisoire à Bicêtre en 1878, puis avait travaillé comme interne avec Vulpian, Cornil et Ranvier. C'est sans aucun doute grâce à la recommandation de ces trois hommes qu'il obtint le poste à la Salpêtrière. Alfred Vulpian (1826-1887), collègue et ami de Charcot, avait fondé avec lui le service de neurologie de l'hôpital et Victor Cornil (1837-1908) fut l'un des premiers internes placés au service du grand neurologue. Quant à Louis Ranvier (1835-1922), il était déjà directeur du laboratoire d'histologie du Collège de France et était respecté dans le milieu scientifique français. En plus de l'appui de ces trois professeurs, Babinski avait déjà publié un article important sur la sclérose en plaques ${ }^{5}$ qui ne manqua pas de faire bonne impression sur Charcot.

Le grand maître, quant à lui, approchait de la soixantaine et se trouvait probablement à l'acmé de sa carrière. Après deux voyages célèbres en 1881 (à Londres et en Russie) qui consolidèrent sa réputation internationale, une chaire clinique pour les maladies du système nerveux fut créée à son intention en 1882 et il fut nommé l'année suivante à l'Académie des Sciences. ${ }^{6}$ Ses articles dans les journaux se multipliaient, ${ }^{7}$ commentant les leçons qu'il donnait à la Salpêtrière, en particulier sur l'hystérie. De plus, Pierre Marie (1853-1940), le chef de clinique auquel devait succéder Babinski, avait eu de très bons rapports avec Charcot, au point de l'inviter à être son témoin lors de son mariage. C'est dans ce contexte que le jeune Parisien d'origine polonaise entre dans le service du grand maître. On peut donc essayer de deviner ses sentiments, probablement empreints d'anxiété, du désir de bien faire mais aussi d'excitation quant à l'enseignement qu'il allait recevoir, sentiments que remplaceraient bientôt le respect et l'amitié développés avec le temps.

En effet, Joseph Babinski fut très vite dans les faveurs du maître. Lorsque Sigmund Freud décrit son expérience à la Salpêtrière, il qualifie Babinski d' «élève préféré du maître». ${ }^{8} \mathrm{~A}$ posteriori, cela peut paraître inévitable. Comme le rappelle clairement Georges Guillain dans la biographie qu'il a écrite sur son maître, celui-ci «ne s'intéressait qu'à ceux de ses élèves qu'il jugeait intelligents, ... il ne s'occupait que de l'élite. Cette élite était pour lui une vraie famille qu'il accueillait avec une

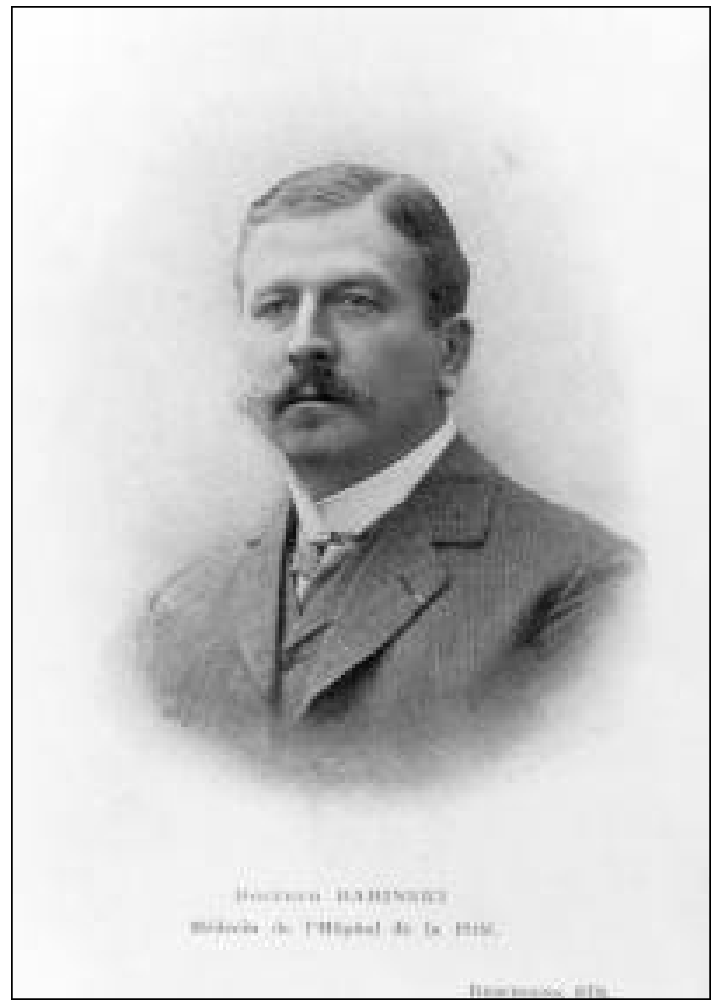

Figure 2: Joseph Babinski (1857-1932). From the Osler Library of the History of Medicine, McGill University, Montreal 
profonde affection à son foyer». ${ }^{9}$ Babinski, ce «géant blond à l'œil bleu» ${ }^{3}$ et à l'intelligence vive, réussit à en faire partie. En effet, dès 1887, le jeune neurologue participe à une représentation de Macbeth pour la Saint-Martin en compagnie de Jean-Martin Charcot, de sa femme, de ses enfants et de deux de ses amis les plus proches. Tel que le rapporte Léon Daudet dans ses Souvenirs, ${ }^{10}$ il est un régulier des soirées du mardi organisées par son patron, où il est décrit de façon élogieuse: «Au premier rang, deux intelligences lumineuses, Brissaud et Babinski.»De plus, en tant que chef de clinique, il figure sur le fameux tableau d'André Brouillet de 1887, Une leçon clinique à la Salpêtrière. On y voit Charcot entouré de tous ses élèves, internes et étudiants, donner un cours sur l'hystérie, avec Babinski à sa gauche supportant, évanouie dans ses bras, une belle patiente (Blanche Wittman). Babinski préfacera lui-même le livre édité en 1888 par trois élèves de Charcot, (Blin, J.-B. Charcot et Colin) où ces derniers ont recueilli les propos du maître durant ses leçons du mardi. Il profite de cette occasion pour en faire la louange, rapportant les propos d'un physicien allemand en visite à Paris, et prendra ainsi une habitude qui ne le quittera jamais, celle de toujours parler de lui avec le plus grand respect, ${ }^{11}$ même lorsque ses idées l'opposaient à celles de son professeur (comme nous le verrons plus loin au sujet de l'hystérie). Enfin, certains prétendent même qu'il était l'homme que Charcot avait en tête pour lui succéder à la chaire de neurologie à la Salpêtrière s'il n'avait pas échoué au concours de l'agrégation, le rendant ainsi inéligible. $^{12}$

\section{ÉCHEC AU CONCOURS DE L’AGRÉGATION DE 1892}

Malheureusement, il y eut une occasion pour laquelle cette amitié ne joua pas en sa faveur. Ainsi qu'il a été signalé plus haut, Charcot traitait comme les membres de sa propre famille ceux parmi ses élèves qu'il jugeait dignes de cet honneur, réussissant par là même à créer une véritable école, solidaire de son maître, et dont chaque membre pouvait se prévaloir d'un fort sentiment d'appartenance. Les membres de cette confrérie s'entraidaient au sein d'une saine compétition tel que le démontre la nomination de Babinski comme chef de clinique sur la simple recommandation de Cornil et Vulpian. L'un des principaux désavantages de ce système reposait évidemment sur le fait qu'il était difficile de se bâtir une carrière académique en neurologie tant que le maître jouissait d'un aussi puissant rayonnement. Ainsi, la majorité des premiers internes et chefs de clinique sous Charcot orientèrent leur carrière vers des domaines connexes, pour ne pas être en concurrence avec le célèbre neurologue; nommons par exemple Victor Cornil, professeur de médecine, Pierre Janet et Alexis Joffroy en psychiatrie, Maurice Debove, futur doyen de la faculté de médecine ... Charles Bouchard (1837-1915), en revanche, fut le seul interne à abandonner Charcot dans un premier temps, avant de le défier ouvertement par la suite. Guillain ${ }^{9}$ a tenté d'expliquer les raisons de cette défection en invoquant le caractère ambitieux des deux hommes. Tous deux, fort intelligents, désiraient fonder leur propre école et avoir leurs propres élèves. C'est ce qui expliquerait pourquoi Bouchard, après avoir été promu au titre de professeur en pathologie générale à Bicêtre, cessa de suivre les conseils de son ancien maître: maintenant qu'il avait ses propres élèves, il les favorisait au détriment de ceux de Charcot.
Ces derniers auraient amplifié la controverse en rapportant de possibles propos de l'un sur l'autre, jusqu' au point culminant du concours de l'agrégation en 1892 . Iragui ${ }^{13}$ retrace en détails les coulisses de cet examen auquel avait postulé Joseph Babinski, en compagnie d'autres étudiants de Charcot, dont le plus connu est probablement Gilles de La Tourette (1857-1904). Bouchard avait été nommé président du jury par le ministre de l'Éducation et certains historiens y avaient vu le premier signe d'une perte de pouvoir politique de la part de Charcot. Les résultats du concours furent dévoilés en mars 1892, avec la nomination de trois élèves de Bouchard et aucun de Charcot. Le premier fut accusé d'avoir poussé les jurés à voter pour ses élèves, en plus d'avoir retardé la première session du concours dans l'intention de garder un des jurés qui lui était favorable. En effet, sans son intervention, Germain Sée (1818-1896) aurait été exclu du jury en raison de son absence lors de la première session à cause d'une colique rénale. Malgré deux recours en appel de la part de Babinski et de trois autres concurrents, la décision fut maintenue et Babinski ne put recevoir l'agrégation cette année-là.

Quelles furent donc les conséquences de cet échec? D'une part, le concours scella pour de bon l'inimitié entre Bouchard et Charcot. Ce dernier, bien que sans l'admettre jamais en public, conserva à l'encontre de son ancien élève une rancœur certaine, d'autant plus que l'affaire fut reprise par la presse et que le public suivit cette controverse comme la preuve d'une altercation de plus entre les deux hommes. La réaction du vieux professeur montre à quel point il estimait son jeune élève. Sa colère peut évidemment s'expliquer en partie par la blessure que venait de subir son orgueil, mais il est certain qu'il fut également très déçu pour son jeune protégé. Ainsi, on ne parle jamais, par exemple, de l'échec de Gilles de La Tourette à ce même concours ni de l'effet que cela eut sur Charcot, mais bien de l'échec de Joseph Babinski.

Quant à Babinski lui-même, après que ses appels eurent été rejetés, il ne repassa plus jamais l'agrégation et fut ainsi privé de toute possibilité de carrière académique. Cette décision semble à première vue assez surprenante. En effet, il n'était pas rare d'échouer lors de sa première tentative au concours de l'agrégation. Charcot lui-même n'avait pas obtenu la sienne en 1857, et avait failli être recalé une deuxième fois lors sa seconde tentative en $1860 .{ }^{6}$ Cependant, l'appui de Rayer à cette époque avait incité le jeune médecin à poursuivre ses efforts. Babinski ne se trouve pas dans la même situation en 1894, lorsque son second appel est rejeté. Ses principaux maîtres et appuis politiques, Charcot et Vulpian, sont décédés depuis peu et il ne connaît aucune personnalité influente susceptible de soutenir sa candidature. Il se peut également qu'étant devenu chef du service de neurologie à la Pitié en 1895, il n'ait plus senti la nécessité d'un titre et donc d'une carrière académique. Finalement, dans ses commentaires sur la traduction des Leçons du mardi, le professeur Goetz avance une hypothèse intéressante. ${ }^{11}$ Il se base sur une caricature, parue dans la revue Chantecler, où Babinski se pointe du doigt en disant: «ceci est mon Cygne». À partir de cette illustration, il émet l'hypothèse d'une possible discrimination sociale qui expliquerait l'existence d'un fossé entre la reconnaissance internationale de Babinski et son manque de titres académiques en France. Le cygne était en effet reconnu comme un symbole de l'hermaphroditisme et la société française de la fin du dix-neuvième siècle voyait d'un mauvais œil le fait 
que Babinski ne se soit jamais marié. Cette théorie n'est cependant étayée par aucune autre référence précise dans aucun article, pamphlet ou caricature de l'époque.

Il reste cependant difficile de déterminer exactement les conséquences de ce concours sur la carrière de Babinski. Beaucoup de ses élèves en ont minimisé l'importance, et ont au contraire insisté sur la liberté que ce manque de responsabilité académique lui conférait pour s'adonner pleinement à la neurologie clinique, dans son service de la Pitié, ainsi qu'à l'enseignement. Cependant, il ne fait aucun doute que ce manque de reconnaissance par ses pairs ne l'empêcha pas de devenir l'une des figures marquantes de la neurologie et d'être sans cesse admiré et respecté par ses élèves. Ainsi en témoigne ce touchant hommage que lui fit Maxime Laignel-Lavastine (1875-1953), lors de sa leçon inaugurale en tant que professeur, en novembre 1931: «Permettez-moi de le saluer ici où il aurait dû naguère enseigner», ${ }^{14}$ faisant référence à la faculté de médecine de Paris.

\section{TraVAuX SUR L'HYSTÉRIE}

Il paraît évident que ces deux grandes personnalités de la neurologie française entretenaient une relation professeur-élève emplie de respect, auquel se mêlait peut-être une certaine forme d'affection filiale de la part de Charcot. Il n'est donc pas surprenant de retrouver l'influence du célèbre professeur dans les premières œuvres de Babinski. Ce dernier est arrivé à la Salpêtrière au moment même où ses recherches sur l'hystérie étaient florissantes. Ses premières publications sont toutes consacrées à l'hystérie. Dans les quatre publications, on retrouve des thèses qui sont en accord avec les travaux de son maître, défendant sa position contre l'école de Nancy, autre centre important de l'époque qui se spécialisait en particulier dans la recherche sur l'hystérie et l'hypnotisme. Ainsi, il soutient que certaines manifestations de l'hystérie peuvent être transmises par l'aimant d'un patient à l'autre; il lutte contre l'idée que certains symptômes, dits somatiques, de l'hystérie puissent être induits par suggestion, réfute que des sujets normaux non hystériques soient possiblement hypnotisables, et cherche à prouver le lien entre hystérie et certains troubles somatiques. ${ }^{4}$ Il est clairement en accord avec le point de vue de Charcot sur l'hystérie.

C'est en mai 1893, quelques mois avant la mort de son maître, que Babinski publie la première observation qui va progressivement révolutionner sa vision de cette maladie. En effet, il note clairement que dans les cas de paralysie hystérique, contrairement aux autres cas de paralysie, «les réflexes tendineux ne sont pas exagérés», ${ }^{4}$ marquant ainsi la première séparation, dans le domaine de l'hystérie, entre signes physiques objectifs et symptômes subjectifs formulés par le patient. Cette préoccupation sera d'ailleurs présente tout au long de sa carrière, et il ne cessera d'en rappeler l'importance à ses élèves: «Les phénomènes objectifs d'une valeur capitale sont ceux que la volonté est impuissante à reproduire. Vous ne sauriez trop, Messieurs, vous appliquer à les étudier.» ${ }^{4}$ Auguste Tournay retrace avec précision les étapes de ce revirement sur l'hystérie. Ainsi, Babinski prétend que «[s]es études poursuivies pendant une dizaine d'années aboutissent en 1901 à la communication à la Société de Neurologie» où il offre sa propre définition de l'hystérie et propose de remplacer ce terme désuet par le néologisme de «pithiatisme», ou guérissable par suggestion.
Cette communication est complètement révolutionnaire, et opère un revirement à cent quatre-vingts degrés par rapport à la position qui était la sienne et celle de son ancien maître. Babinski y affirme que l'hystérie n'est que le produit de la suggestion, qu'elle soit autosuggestion, suggestion sociale ou suggestion par hypnotisme, et que par conséquent, elle est immédiatement guérissable par la persuasion. Il se dit prêt à défendre ses idées et reste ouvert à toute discussion sur le sujet, comme il ne fait aucun doute dans son esprit que les protestations ne vont pas tarder à se faire entendre. C'est finalement au congrès de Lausanne, en 1908, que sont lancées les principales attaques, et que Babinski va clarifier sa position en la réaffirmant. Les années qui suivront et la dure épreuve de la guerre viendront confirmer sa thèse, et voir progressivement disparaître l'hystéro-épilepsie ou grande hystérie de Charcot. ${ }^{6}$ Les exemples abondent pour prouver que des patients imitaient les épileptiques avec qui ils étaient regroupés dans la même salle, et étaient ainsi victimes de suggestion. La guérison de ces hystéro-épileptiques survenait dès qu'on les séparait des épileptiques, dès qu'on cessait de s'intéresser à leur cas ou encore, pour un certain nombre d'entre eux, à la mort de Charcot, lorsqu'ils sentirent que leur cas n'intéressait plus ses successeurs. Ainsi, en janvier 1912, l'on peut citer le professeur Chauffard qui affirme: «il n'y a presque plus d'hystérie dans nos services.» ${ }^{4}$

Ce revirement peut ressembler à une traitrise de Babinski à l'égard de Charcot, mais tous, et Babinski le premier, se sont efforcés de nous démontrer qu'il ne faisait que continuer le travail du maître et qu'il ne lui adressait aucun reproche direct. Ainsi, même après avoir complètement révisé sa vision de l'hystérie, il reprend la notion d'hystérie traumatique, que Charcot avait mise de l'avant bien des années auparavant, et développe certains des travaux de son maître. ${ }^{6}$ De même, Babinski rappelle que «si les résultats de mes recherches m'ont conduit à abandonner la doctrine de mon illustre maître, je n'en conserve pas moins ... une admiration profonde pour le grand neurologiste», comme il le confirmera d'ailleurs dans un touchant éloge funèbre. ${ }^{15}$ Ainsi, même lorsqu'il remet en question une grande partie de l'œuvre de son maître, Babinski cherche toujours à le faire dans le respect de ce dernier, et s'efforce de garder sa renommée intacte. Enfin, certains indices peuvent même nous laisser croire que le point de vue de Charcot, avant sa mort, commençait à abonder dans le sens de son élève, et que ce dernier était sur le point de le convaincre du bien-fondé de ses arguments.

En premier lieu, dans un travail publié en 1893 et supervisé par Charcot, Freud conclut, en contradiction totale avec les croyances en une lésion anatomique de Charcot, que l' «hystérie se comporte, dans ses paralysies et autres manifestations, comme si l'anatomie n'existait pas.» Deuxièmement, dans la préface au livre de Janet, son ancien interne, que Charcot écrivit en 1892, il souligne le rôle grandissant et l'importance de la psychologie dans la compréhension de l'hystérie. ${ }^{6}$ Finalement, dans son tout dernier ouvrage, La foi qui guérit, une citation laisse présager que si la mort ne l'en avait pas empêché, il se serait probablement joint aux vues de son disciple: «Les hystériques possèdent un état mental éminemment favorable au développement de la Faith healing, car ils sont suggestibles au premier chef, soit que la suggestion s'exerce par des influences extérieures, soit surtout qu'ils présentent en eux-mêmes les 
éléments si puissants de l'auto-suggestion. Chez ces individus, l'influence de l'esprit sur le corps est assez efficace pour produire la guérison des maladies que l'ignorance où on était, il n'y a pas longtemps encore, de leur nature véritable, faisaient considérer comme incurables.» ${ }^{16}$ Georges Guinon, le dernier secrétaire particulier de Charcot, le confirme d'ailleurs en rapportant une conversation qu'il avait eue avec lui, où il disait que «notre conception de l'hystérie était devenue caduque, qu'il allait falloir bouleverser tout ce chapitre de la pathologie nerveuse», annonçant en quelque sorte le travail futur de Babinski. $^{9}$

\section{UNE RESSEMBLANCE ÉVIDENTE}

En étudiant les nombreux témoignages des disciples de ces deux grands neurologues, l'on ne peut s'empêcher de s'attarder sur certaines similitudes entre les deux hommes. Quelques coïncidences amènent d'abord le sourire, par exemple le fait que Babinski ait passé toute sa carrière à l'hôpital de la Pitié, où ses deux maîtres, Charcot et Vulpian, se rencontrèrent pour la première fois et nouèrent amitié durant leur internat; ou encore qu'il mourut des conséquences de la maladie de Parkinson, alors que son maître en fut également atteint, ${ }^{17}$ lui qui l'avait auparavant décrite en détail et avait distingué son tremblement du tremblement sénile bénin. Remarquons également que tous deux eurent des homonymes contemporains célèbres, Charcot dans la personne de son fils, Jean-Baptiste, ${ }^{18}$ neurologue comme lui, mais qui se distingua en tant qu'explorateur du Pôle Nord, et Babinski dans la personne de son frère Henri, connu du ToutParis comme fin cuisinier, sous le pseudonyme d'Ali-Bab. ${ }^{19}$ Mais ce qui est surtout frappant, à lire les œuvres de leurs étudiants, est la façon presque identique avec laquelle ils dépeignent les deux hommes conduisant l'examen clinique des patients devant leurs étudiants, dans un silence absolu, sans prononcer un mot, et arrivant au diagnostic sous l'œil ébahi de leurs disciples.

\section{UNE RELATION HIPPOCRATIQUE}

Il paraît donc logique de penser que Charcot eut une influence décisive sur toute la vie et la méthode médicale de Joseph Babinski. Dès son entrée à la Salpêtrière, suite aux recommandations d'anciens internes du grand maître, ce dernier reconnut en lui une intelligence brillante et le prit immédiatement sous son aile. Il l'aida dans ses travaux de recherche, lui procura un modèle d'enseignement constant, mais surtout il l'introduisit dans le fameux cercle de l'École de la Salpêtrière dont Babinski gardera la marque toute sa vie. Le concours de l'agrégation de 1891, qui opposa les influences politiques de Charcot et Bouchard, et dont Babinski fut la victime, démontre à quel point le grand maître tenait ce jeune neurologue en estime et en affection, de par la profonde déception qu'il ressentit suite à son échec. Babinski, de son côté, lui fut toujours reconnaissant, et même lorsqu'il eut à le contredire, comme dans le cas de l'hystérie, il fit montre à son égard du plus grand respect et de la plus grande admiration. Ce dévouement constant de l'élève envers le maître, même après la mort de celui-ci, n'est pas sans rappeler les principes d'Hippocrate qui demandait de la part de ses élèves un respect et un amour du maître, dans une relation qui allait bien au-delà du simple rapport professionnel et qui relevait presque d'un lien filial. C'est exactement de la même façon que, beaucoup plus tard, Babinski traita ses propres élèves, qui eux aussi lui témoignèrent une admiration et un respect sans bornes.

\section{RÉFÉRENCES}

1. McHenry LC. Garrison's History of Neurology. Springfield, Illinois: Charles C Thomas Publisher, 1969.

2. Van Gijn. The Babinski Sign, a Centenary. Universiteit Utrecht, 1996.

3. Charpentier A. Un grand médecin, Joseph Babinski, (1857-1932). Paris: La typographie François Bernouard Dr, 1934.

4. Tournay A. La vie de Joseph Babinski. Amsterdam: Elsevier Publishing Company, 1967.

5. Babinski J. Étude anatomique et clinique sur la sclérose en plaques. Paris, Masson, 1885.

6. Bonduelle M, Gelfand T, Goetz CG. Charcot: un grand médecind dans son siècle. New York: Oxford University Press, 1995.

7. Thuillier J. Monsieur Charcot de la Salpêtrière. Paris: Éditions Robert Laffont, 1993.

8. Freud S. Pre-psychoanalytic Publications and Unpublished Drafts. Strachey J, Freud A (Eds.) London, Hogarth, Toronto, Irwin, 1966, vol. 1 (1886-1899).

9. Guillain G. J-M Charcot, sa vie, son œuvre. Paris: Masson et Cie, 1955.

10. Daudet L. Souvenirs I. Paris: Nouvelle Librairie Nationale, 1915.

11. Goetz CG. Charcot, The Clinician, The Tuesday Lessons. New York: Raven Press, 1987.

12. Satran R. Fulgence Raymond, the successor of Charcot. Bull N Y Acad Med. 1974;50(8):931-942.

13. Iragui V. The Charcot-Bouchard controversy. Arch Neurol 1986;43:290-295.

14. Dartigues L. Faisceau scriptural, vol. 3. Paris: G. Dom, 1932.

15. Babinski J. Éloge de J.-M. Charcot. Revue neurologique I, 6:745756.

16. Charcot J-M. La foi qui guérit. The faith healing. Revue hebdomadaire. Paris: Plon, 1892.

17. Goetz CG. Charcot and the aging brain. Arch Neurol 2002;59:18211824.

18. Kyle R, Shampo M. Jean-Baptiste-Étienne-Auguste Charcot. JAMA1984; 252(2):257.

19. Gasecki A. Hachinski V. On the names of Babinski. Can J Neurol Sci 1996; 23: 76-79. 